\title{
Transcription, torsional stress and the bending dynamics of DNA
}

\begin{abstract}
In this research, we found that the local opening of base pairs induces the formation of kinks which facilitates the bending of double helix. The conformational properties of DNA can be mapped onto the Heisenberg spin system and denaturation occurs through quantum phase transition (QPT) induced by a quench when the temperature effect is incorporated through the quench time. The nonequilibrium effect in QPT introduced through the quench generate defects like kinks end antikinks, the density of which depends on the quench time and hence on temperature. It is here argued that when we transcribe this result in the rod-like-chain (RLC) model of DNA, these defects correspond to bends. The dynamical formation of these bends during local denaturation associated with transcription hinders free rotation of the transcribed DNA and helps the torsional stress to propagate down the DNA. This explains the observed large torsional stress near the point of transcription. We have estimated the bend length which is found to be in good agreement with experiments.
\end{abstract}

PACS numbers: 87.15-v 63.20.Pw 87.14gk.

Keywords: base pairs, double helix, Heisenberg spin system, quantum phase transition, torsional stress
Volume 4 Issue I - 2020

\author{
Subhamoy Singha Roy \\ Department of Physics, JIS College of Engineering \\ (Autonomous),West Bengal University of Technology, India
}

Correspondence: Subhamoy Singha Roy, Department of Physics, JIS College of Engineering (Autonomous), West Bengal University of Technology, Kalyani, Nadia -74I235, India, Emailssroy.scienc@gmail.com

Received: September 08, 2019 | Published: February 17, 2020
Abbreviations: QPT, quantum phase transition; RLC, rod like-chain; QPT, quantum phase transition; KZ, kibble-zurek; WLC, worm-like-chain

\section{Introduction}

It is well known that transcription induces torsional stress in DNA. Indeed the transport of torsional stress (torque) along DNA during transcription affects the activity of nearby genes. Transcription causes axial rotation of the transcripted DNA relative to the transcribing polymerase. If the rotation is hindered in any way torsional stress will propagate along the DNA disturbing the stability of the double helix structure at some point. In a viscous medium a straight infinite rod experiences a frictional resistance to axial rotation given by

$$
\tau=\mu L
$$

where $\tau$ is the torque with dimension of energy, $L$ is the length and $\mu$ represents the friction constant. Liu and Wang ${ }^{1}$ pointed out that the friction constant is very small and no significant torsional stress was possible in DNA of reasonable length without any other physical anchoring However experimental observations, both in living cells as well as in vitro suggest that there are significant torsional stress after transcription at a single promoter on unanchored DNA. In a seminal paper Nelson ${ }^{2}$ resolved this paradox by pointing out that DNA is a heteropolymer and is naturally bent on length scales larger than its persistent length of about $50 \mathrm{~nm}$. The natural bends resist the flexing which is required for a curved molecule to spin in place without dragging sideways through the surrounding medium. This resistance to flexing increases the viscous drag through the surrounding water. The enhanced drag explains the long torsional stress near the point of transcription. Nelson ${ }^{2}$ considered that natural DNA is a stack of similar bent nonidentical subunits arranged in an order which is random .It has been assumed that though these bends are random their effects do not average to zero on length scales much longer than one base pair.

\section{Theoretical background}

The Hamiltonian of the one dimensional Heisenberg spin chain (XXX model), with nearest neighbour interaction is given by

$$
H=\sum_{i} \sigma_{i}^{X} \sigma_{i+1}^{X}+\sigma_{i}^{Y} \sigma_{i+1}^{Y}+\sigma_{i}^{Z} \sigma_{i+1}^{Z}+\lambda \sum_{i} \sigma_{i}^{2}
$$

$\sigma_{i}^{X(Y, Z)}$ is the pauli matrix at the site $i$, which represents the spin vector through the rotation $\vec{S}=(1 / 2) \vec{\sigma}$ and $\lambda$ is the parameter representing the external magnetic field. In the context of DNA, $\lambda$ represents torsion energy. ${ }^{3}$ When the spin system undergoes quantum phase transition (QPT) the criticality corresponds to a two-limit behaviour. At $\lambda=0$ the system represents the antiferromagnetic state and at $\lambda=2$ the system represents the ferromagnetic state. The region between $\lambda=0$ and $\lambda=2$ represents the critical region. The entanglement entropy is maximum at $\lambda=0$ and it decreases as $\lambda$ increases when at $\lambda=2$ it vanishes. It is now observed that the criticality of a finite temperature transition can be mapped onto a zero temperature QPT induced by a quench as the nonequilibrium effect in zero temperature QPT expresses a remarkable analogy between phase transition at finite and zero temperature. ${ }^{5}$

In fact quantum criticality can be approached in two different ways. We may view that as $T \rightarrow 0$ the control parameter $\lambda$ attains the critical value $\lambda=\lambda_{c}$.Also it may be viewed that at $T=0$, $\lambda \rightarrow \lambda_{c}{ }^{8}$ As the critical point is approached the spatial correlation as well as the temporal correlation of the order parameter fluctuations become long ranged. The typical time scale for the decay of the fluctuations is the correlation time which diverges near criticality. The crossover form the quantum to classical behaviour will occur when the 
correlation time exceeds $\beta=1 / k_{B} T$ in a quench induced QPT. This happens when we introduce the Kibble -Zurek (KZ) mechanism ${ }^{7,8}$ in QPT taking into account time dependent control parameter which gives rise to nonequilibrium effect near criticality. This implies that zero temperature QPT induced by a quench can be mapped onto a classical finite temperature phase transition when temperature effect is incorporated in the quench time $\tau_{q}$ having the relation $T \sim 1 / \tau_{q}$. In QPT induced by a quench in Heisenberg spin chain we can take

$$
\lambda(t<0)=2-2 t / \tau_{q}
$$

so that at $t=\tau_{q}$ we have $\lambda=0$ and as $t$ approaches zero starting from $\tau_{q}, \lambda$ increases so that at $t=0, \lambda=2$ when the entanglement entropy vanishes. It has been shown in a recent paper $^{9}$ that in the Heisenberg spin chain the $\lambda$ dependence of the entanglement entropy $S$ is given by

$$
S(\lambda)=S(\lambda=0)+\Delta S
$$

with $\Delta S=(1 / 6) \log _{2}(1-\lambda / 2)$

It may be mentioned here that in studying denaturation of DNA in terms of QPT induced by a quench the temperature dependence $\lambda$ of is taken to be ${ }^{3}$

$$
\lambda=\bar{A} T+\bar{B}
$$

where $\bar{A}$ and $\bar{B}$ are constants given by $\bar{A}=0.0385$ and $\bar{B}=-11.47$

From eqns $(4,5,6)$ we can now determine the entropy at a given temperature which gives a measures of the opening of base pairs at a given temperature. ${ }^{4} \mathrm{~A}$ general property of QPT induced by a quench is that it generates defects in the form of kinks and antikinks and introduces a new length scale known as the Kibble -Zurek (KZ) correlation length defined by the average distance between a kink and the nearest antikink. ${ }^{10,11}$ The density of defects $\rho$ depends on the quench time $\tau_{q}$ through the relation $\rho \sim 1 / \sqrt{\tau_{q}}$ so that the $\mathrm{KZ}$ correlation length $\xi$ satisfies the relation $\xi \sim \sqrt{\tau_{4}} .{ }^{10,11}$ Utilising the relationship between the quench time $\tau_{q}$ with temperature $T$ through the relation $T \sim 1 / \tau_{q}$ as mentioned above, we note that the $\mathrm{KZ}$ correlation length $\xi$ depends on the temperature through the relation $1 / \sqrt{k_{B} T}$.

The Worm-like-chain (WLC) model: It is noted that the elastic properties of DNA can be best formulated when it is considered as an elastic object. The Worm-like-chain (WLC) model was introduced $^{12}$ which describes a chain by elastic continuous curve at thermal equilibrium with a single elastic constant, the persistent length $\mathrm{P}$, characterizing the bending energy. The WLC can be solved analytically by mapping it onto a quantum mechanical problem. Indeed the partition function is a Euclidean path integral for a quantum dumbbell. Bouchiat and Mezard ${ }^{13}$ generalized this model introducing twist rigidity and it appears that a DNA molecule can be depicted as a thin elastic rod. The rod-like-chain (RLC) model is characterized by the fact that the partition function can be mapped onto the path integral representing the charged particle in the field of a nonquantized magnetic monopole.

Now we transcribe our above results derived from the situation where a double helix is mapped onto the Heisenberg spin chain into the elastic rod model. It is noted that local denaturation associated with transcriptions caused by QPT induced by a quench leads to the generation of defects like kinks and antikinks which correspond to the bends of the axis of the DNA molecule and represent dynamical bending of the elastic rod. The KZ correlation length corresponds to the bend length. These bends are dynamically produced and the associated bend length $A$ is different from the structural persistent length $\mathrm{P}$ which is the geometrical property of the rod. As the formation of the defects is such that a kink is followed by an antikink, this would mean that every joint periodically bends oppositely to each other. From the relation that the $\mathrm{KZ}$ correlation length $\xi \sim \sqrt{\tau_{q}}$ and $\tau_{q} \sim$ $1 / T$ we note that when we consider $\xi$ as the bend length $A$, we can write the relation $A \sim 1 / \sqrt{T}$. From this we write

$$
\left(A^{2} / P\right)=k / K_{B} T
$$

Where $k$ is the bend stiffness having dimension of energy.length. Here we have introduced the structural persistent length P in the L.H.S for dimensional reason as this corresponds to a fixed length scale for the system. Now observing that experimentally we have bend stiffness $k=45 \mathrm{~nm}\left(K_{B} T\right)$ and $P=130 \mathrm{~nm}^{14}$ we have $A=76.5 \mathrm{~nm}$.This is to be compared with the experimental value $80 \mathrm{~nm}^{14,15}$ From this we have the total persistence length given by

$$
A_{\text {tot }}=\left(A^{-1}+P^{-1}\right)^{-1}
$$

From eqn. (8) we have $A_{t o t}=48.3 \mathrm{~nm}$ which is to be compared with the standard value $50 \mathrm{~nm}$. There we find that our results are consistent with experiments.

\section{Discussion}

It may be recalled here that Nelson ${ }^{2}$ proposed that natural bends in DNA hinder the axial rotation of the transcribed DNA causing the propagation of torsional stress. The bends are taken to be random bunt their effects do not average to zero. In contrast the present analysis suggests that bends are produced dynamically during local denaturation associated with transcription. These bends follow a specific pattern having a more or less same distance between them determined by the $\mathrm{KZ}$ correlation length so that the bend length $A$ satisfies the eqn (7). According to Nelson's prescription the total effect of random natural bends makes DNA a random coil with total persistence length $A_{t o t}$. However according to the present formulation the bends are produced dynamically which make DNA a systematic coil having a well-defined bend length and total persistence length.

Nelson ${ }^{2}$ proposed a hybrid model for DNA selecting a crossover length $l_{c}$ and executing a hybrid motion. In length scales shorter than $l_{c}$ the motion is nearly rigid which resists flexing. On longer length scales the motion crosses over to spinning (plumber's snake) as rigid (crankshaft) motion has a large viscous drag in length scales. A random bent rod has structure on every length scale and the drag torque per length increases without bound for longer segments until the crossover length $l$ is reached. Since $l$ is estimated to be about $1.4 \mathrm{kbp}^{13}$ cramped DNA is effectively spin locked on scales shorter than $l_{c}$ and explains the large torsion stress near the point of transcription. In our present formalism we observe that when the conformational properties of a DNA molecule associated with the Heisenberg spin system is mapped onto the elastic rod model, bends are formed dynamically during transcription and the segments between any two bends undergo rigid motion which resist flexing. ${ }^{16,17}$ Indeed the formation of bends during local denaturation associated with the transcription process effectively corresponds to defects like 
kinks and antikinks when we consider the DNA molecules as a spin system undergoing QPT induced by a quench and these defects hinder the axial rotation of the transcripted DNA. This explains the large observed torsional stress near the point of transcriptions.

\section{Summary}

In summary, we argue that local denaturation during transcription leads to the generation of defects like kinks and antikinks which correspond to bend in the elastic rod formulation of DNA. This dynamical formation of bends shut down spinning motion completely and generates significant torsional stress near the point of transcription.

\section{Acknowledgments}

None.

\section{Conflicts of interest}

The author declares there is no conflict of interest.

\section{Funding}

None.

\section{Reference}

1. L Liu, J Wang. Supercoiling of the DNA template during transcription. Proc Natl Acad Sci (USA). 1987;84;7024-7027.

2. P Nelson. Transport of torsional stress in DNA. Proc Natl Acad Sci (USA). 1999;96:14342-14347.

3. S Singha Roy, P Bandyopadhyay. Bending Dynamics of DNA. Phys Lett A. 2013;337:2884.

4. S Singha Roy, P Bandyopadhyay. Entropy-driven denaturation and bubble nucleation in DNA melting. Europhysics Lett. 2015;109:48002.

5. R Schutzhold. Quantum back reaction in dilute Bose-Einstein condensates. Phys Rev Lertt. 2005;95:133703.
6. M Vojta. Quantum phase transitions. Rep Prog phys. 2013;66:2009.

7. TWB Kibble. Topology of cosmic domains and strings. J Phys A Math Gen. 1976;9:1387.

8. WH Zurek. Cosmological Experiments in Condensed Matter Systems. Phys Rep. 1996;276:177(1996).

9. B Basu, P Bandyopadhyay, P Majumder. Magnetic-field dependence of the entanglement entropy of one-dimensional spin systems in quantum phase transitions induced by a quench. Phys Rev A. 2012;86:022303.

10. L Cincio, Jacek Dziaemaga, Marek M, et.al. Entropy of entanglement and correlations induced by a quench: Dynamics of a quantum phase transition in the quantum Ising model. Phys Rev A. 2007;75:052321.

11. B Basu, P Bandyopadhyay, P Majumder. Density of defects and the scaling law of the entanglement entropy in quantum phase transition of one-dimensional spin systems induced by a quench. Phys Rev A. 2011;83:032312.

12. C Bustamante, JF Marko, ED Siggia, et al. Entropic elasticity of lambdaphage DNA. Science. 1994;265(5178):1599-1600.

13. C Bouchiat, M Mezard. Elasticity Model of a Supercoiled DNA Molecule. Phys Rev Lett. 1997;80:1556.

14. J Bednar, Furrer P, Katritch V, et al. Determination of DNA persistence length by cryo-electron microscopy. Separation of the static and dynamic contributions to the apparent persistence length of DNA. J Mol Biol. 1995;254(4):579-594.

15. S Singha Roy. DNA Molecule as a Spin System and the Symmetric Top Model. Theoretical Physics. 2017;2(3):141.

16. S Singha Roy, P Bandyopadhyay. Quantum perspective on the localized strand separation and cyclization of DNA double helix. Phys Lett A. 2018;382:973.

17. B Basu, P Bandyopadhyay, P Majumdar. Magnetic-field dependence of the entanglement entropy of one-dimensional spin systems in quantum phase transitions induced by a quench. Phys Rev A. 2012;86:022303. 